\title{
Some New Traveling Wave Solutions of the Nonlinear Reaction Diffusion Equation by Using the Improved $\left(G^{\prime} / G\right)$-Expansion Method
}

\author{
Hasibun Naher and Farah Aini Abdullah \\ School of Mathematical Sciences, Universiti Sains Malaysia, 11800 Penang, Malaysia \\ Correspondence should be addressed to Hasibun Naher, hasibun06tasauf@gmail.com \\ Received 5 December 2011; Revised 23 February 2012; Accepted 24 February 2012 \\ Academic Editor: Jun-Juh Yan \\ Copyright (C) 2012 H. Naher and F. A. Abdullah. This is an open access article distributed under \\ the Creative Commons Attribution License, which permits unrestricted use, distribution, and \\ reproduction in any medium, provided the original work is properly cited. \\ We construct new exact traveling wave solutions involving free parameters of the nonlinear \\ reaction diffusion equation by using the improved $\left(G^{\prime} / G\right)$-expansion method. The second-order \\ linear ordinary differential equation with constant coefficients is used in this method. The obtained \\ solutions are presented by the hyperbolic and the trigonometric functions. The solutions become \\ in special functional form when the parameters take particular values. It is important to reveal that \\ our solutions are in good agreement with the existing results.
}

\section{Introduction}

Nonlinear evolution equations (NLEEs) describe many problems of solid state physics, nonlinear optics, plasma physics, fluid mechanics, population dynamics and many others which arise in mathematical biology, engineering sciences and other technical arena. In recent years, several methods have been developed to obtain traveling wave solutions for many NLEEs, such as the theta function method [1], the Jacobi elliptic function expansion method [2], the Hirota's bilinear transformation method [3], the F-expansion method [4], the Backlund transformation method [5, 6], the generalized Riccati equation method $[7,8]$, the sub-ODE method [9], the homogeneous balance method [10,11], the tanh-coth method [1214], the sine-cosine method [15], the first integral method [16], the Cole-Hopf transformation method [17], the Exp-function method [18-25], and others [26-43].

Recently, Wang et al. [44] presented the $\left(G^{\prime} / G\right)$-expansion method and implemented to four well-established equations for constructing traveling wave solutions. In this method, the second-order linear ordinary differential equation (ODE) $G^{\prime \prime}+\lambda G^{\prime}+\mu G=0$ is used, 
where $\lambda$ and $\mu$ are arbitrary constants. Afterwards, many researchers used this method to many nonlinear partial differential equations and obtained many new exact traveling wave solutions. For instance, Malik et al. [45] applied the $\left(G^{\prime} / G\right)$-expansion method for getting traveling wave solutions of some nonlinear partial differential equations. Bekir [46] concerned about this method to study nonlinear evolution equations for constructing wave solutions. Zayed [47] investigated the higher-dimensional nonlinear evolution equations by using the same method to get solutions. In [48], Naher et al. implemented the method for constructing abundant traveling wave solutions of the Caudrey-Dodd-Gibbon equation. Lately, Hayek [49] extended the method called extended $\left(G^{\prime} / G\right)$-expansion method to obtain exact analytical solutions to the KdV Burgers equations with power-law nonlinearity whilst Guo and Zhou [50] expand the method and applied to the Whitham-Broer-Kaup-Like equations and Coupled Hirota-Satsuma KdV equations to construct traveling wave solutions. Zayed and Al-Joudi [51] concerned about the method to find solutions of the NLPDEs in mathematical physics and so on.

More recently, Zhang et al. [52] extended the method which is called the improved $\left(G^{\prime} / G\right)$-expansion method for constructing abundant traveling wave solutions of the nonlinear evolution equations. Then, many researchers implemented the method to construct exact solutions. For example, Hamad et al. [53] solved the higher-dimensional potential YTSF equation by using this powerful and useful method for getting many new exact solutions. In [54], Nofel et al. investigated the higher-order KdV equation via the same method while Zhao et al. [55] applied this method to obtain traveling wave solutions for the variant Boussinesq equations. Tao and Xia [56] executed the method for searching exact solutions of the $(3+1)$ dimensional $\mathrm{KdV}$ equation and so on.

Many researchers studied the nonlinear reaction diffusion equation to obtain traveling wave solutions by using different methods. For instance, Zayed and Gepreel [57] used the $\left(G^{\prime} / G\right)$-expansion method to solve this equation. To the best of our knowledge, the nonlinear reaction diffusion equation is not investigated by using the improved $\left(G^{\prime} / G\right)$-expansion method.

In this paper, we apply the improved $\left(G^{\prime} / G\right)$-expansion method to construct new exact traveling wave solutions of the nonlinear reaction diffusion equation which is very important equation in mathematical biology.

\section{Explanation of the Improved $\left(G^{\prime} / G\right)$-Expansion Method}

Suppose the general nonlinear partial differential equation:

$$
P\left(u, u_{t}, u_{x}, u_{x t}, u_{t t}, u_{x x}, \ldots\right)=0
$$

where $u=u(x, t)$ is an unknown function. $P$ is a polynomial in $u=u(x, t)$ and the subscripts indicate the partial derivatives.

The main steps of the improved $\left(G^{\prime} / G\right)$-expansion method [52] are as follows.

Step 1. Consider the traveling wave variable:

$$
u(x, t)=v(\eta), \quad \eta=x-V t,
$$


where $V$ is the speed of the traveling wave. Using (2.2), (2.1) is converted into an ordinary differential equation for $u=v(\eta)$ :

$$
Q\left(v, v^{\prime}, v^{\prime \prime}, \ldots\right)=0
$$

where the superscripts stand for the ordinary derivatives with respect to $\eta$.

Step 2. Suppose that the traveling wave solution of (2.3) can be presented in the following form [52]:

$$
v(\eta)=\sum_{j=-m}^{m} a_{j}\left(\frac{G^{\prime}}{G}\right)^{j},
$$

where $G=G(\eta)$ satisfies the second-order linear ODE:

$$
G^{\prime \prime}+\lambda G^{\prime}+\mu G=0
$$

where $a_{j}(j=0, \pm 1, \pm 2, \ldots, \pm m), \lambda$, and $\mu$ are constants.

Step 3. To determine the integer $m$, substitute (2.4) along with (2.5) into (2.3) and then take the homogeneous balance between the highest-order derivatives and the highest-order nonlinear terms appearing in (2.3).

Step 4. Substitute (2.4) together with (2.5) into (2.3) with the value of $m$ obtained in Step 3. Equating the coefficients of $\left(G^{\prime} / G\right)^{r},(r=0, \pm 1, \pm 2, \ldots)$, then setting each coefficient to zero, yields a set of algebraic equations for $a_{j}(j=0, \pm 1, \pm 2, \ldots, \pm m), V, \lambda$, and $\mu$.

Step 5. Solve the system of algebraic equations with the aid of commercial software Maple and we obtain values for $a_{j}(j=0, \pm 1, \pm 2, \ldots, \pm m), V, \lambda$, and $\mu$. Then, substituting obtained values in (2.4) along with (2.5) with the value of $m$, we obtain exact traveling wave solutions of (2.1).

\section{Applications of the Method}

In this section, we investigate the nonlinear reaction diffusion equation by applying the improved $\left(G^{\prime} / G\right)$-expansion method for constructing exact traveling wave solutions. 


\subsection{The Nonlinear Reaction Diffusion Equation}

In this work, we consider the nonlinear reaction diffusion equation involving parameters followed by Zayed and Gepreel [57]:

$$
u_{t t}+\alpha u_{x x}+\beta u+\gamma u^{3}=0
$$

where $\alpha, \beta$, and $\gamma$ are nonzero constants. ODE:

Using the traveling wave transformation Equation (2.2), (3.1) is transformed into the

$$
\left(\alpha+V^{2}\right) v^{\prime \prime}+\beta v+\gamma v^{3}=0
$$

where the superscripts indicate the derivatives with respect to $\eta$.

Taking the homogeneous balance between $v^{\prime \prime}$ and $v^{3}$ in (3.2), we obtain $m=1$. Therefore, the solution of (3.2) is in the form as following:

$$
v(\eta)=a_{-1}\left(G^{\prime} / G\right)^{-1}+a_{0}+a_{1}\left(G^{\prime} / G\right),
$$

where $a_{-1}, a_{0}$, and $a_{1}$ are all constants to be determined.

Substituting (3.3) together with (2.5) into the (3.2), the left-hand side of (3.2) is converted into a polynomial of $\left(G^{\prime} / G\right)^{r}(r=0, \pm 1, \pm 2, \ldots)$. According to Step 4 , collecting all terms with the same power of $\left(G^{\prime} / G\right)$ and setting each coefficient of this polynomial to zero yield a set of algebraic equations (which are omitted to display, for simplicity) for $a_{-1}, a_{0}, a_{1}, V, \lambda$, and $\mu$.

Solving the system of obtained algebraic equations with the aid of algebraic software Maple, we obtain the following.

Case 1. One has

$$
a_{-1}=0, \quad a_{0}=\mp \lambda \sqrt{\frac{-\beta}{\gamma\left(\lambda^{2}-4 \mu\right)}}, \quad a_{1}= \pm 2 \sqrt{\frac{-\beta}{\gamma\left(\lambda^{2}-4 \mu\right)}}, \quad V= \pm \sqrt{\frac{2 \beta}{\lambda^{2}-4 \mu}-\alpha},
$$

where $\alpha, \beta$, and $\gamma$ are nonzero constants and $\lambda^{2}-4 \mu \neq 0$.

Case 2. One has

$$
a_{-1}= \pm 2 \mu \sqrt{\frac{-\beta}{\gamma\left(\lambda^{2}-4 \mu\right)}}, \quad a_{0}=\mp \lambda \sqrt{\frac{-\beta}{\gamma\left(\lambda^{2}-4 \mu\right)}}, \quad a_{1}=0, \quad V= \pm \sqrt{\frac{2 \beta}{\lambda^{2}-4 \mu}-\alpha},
$$

where $\alpha, \beta$, and $\gamma$ are nonzero constants and $\lambda^{2}-4 \mu \neq 0$. 
Substituting the general solution Equation (2.5) into (3.3), we obtain two different families of traveling wave solutions of (3.2).

Family 1 (Hyperbolic Function Solutions). When $\lambda^{2}-4 \mu>0$, we obtain

$$
\begin{aligned}
v(\eta)= & a_{-1}\left(\frac{-\lambda}{2}+\frac{1}{2} \sqrt{\lambda^{2}-4 \mu} \frac{A \sinh (1 / 2) \sqrt{\lambda^{2}-4 \mu} \eta+B \cosh (1 / 2) \sqrt{\lambda^{2}-4 \mu \eta}}{A \cosh (1 / 2) \sqrt{\lambda^{2}-4 \mu} \eta+B \sinh (1 / 2) \sqrt{\lambda^{2}-4 \mu}}\right)^{-1}+a_{0} \\
& +a_{1}\left(\frac{-\lambda}{2}+\frac{1}{2} \sqrt{\lambda^{2}-4 \mu} \frac{A \sinh (1 / 2) \sqrt{\lambda^{2}-4 \mu} \eta+B \cosh (1 / 2) \sqrt{\lambda^{2}-4 \mu} \eta}{A \cosh (1 / 2) \sqrt{\lambda^{2}-4 \mu} \eta+B \sinh (1 / 2) \sqrt{\lambda^{2}-4 \mu} \eta}\right.
\end{aligned}
$$

Various known solutions can be rediscovered, if $A$ and $B$ take particular values.

For example:

(i) if $A=0$ but $B \neq 0$, we obtain

$$
\begin{aligned}
v(\eta)= & a_{-1}\left(\frac{-\lambda}{2}+\frac{1}{2} \sqrt{\lambda^{2}-4 \mu} \operatorname{coth} \frac{1}{2} \sqrt{\lambda^{2}-4 \mu \eta}\right)^{-1}+a_{0} \\
& +a_{1}\left(\frac{-\lambda}{2}+\frac{1}{2} \sqrt{\lambda^{2}-4 \mu} \operatorname{coth} \frac{1}{2} \sqrt{\lambda^{2}-4 \mu \eta}\right)
\end{aligned}
$$

(ii) if $B=0$ but $A \neq 0$, we obtain

$$
\begin{aligned}
v(\eta)= & a_{-1}\left(\frac{-\lambda}{2}+\frac{1}{2} \sqrt{\lambda^{2}-4 \mu} \tanh \frac{1}{2} \sqrt{\lambda^{2}-4 \mu} \eta\right)^{-1}+a_{0} \\
& +a_{1}\left(\frac{-\lambda}{2}+\frac{1}{2} \sqrt{\lambda^{2}-4 \mu} \tanh \frac{1}{2} \sqrt{\lambda^{2}-4 \mu \eta}\right)
\end{aligned}
$$

(iii) if $A \neq 0, A>B$, we obtain

$$
\begin{aligned}
v(\eta)= & a_{-1}\left(\frac{-\lambda}{2}+\frac{1}{2} \sqrt{\lambda^{2}-4 \mu} \tanh \left(\frac{1}{2} \sqrt{\lambda^{2}-4 \mu} \eta+\eta_{0}\right)\right)^{-1}+a_{0} \\
& +a_{1}\left(\frac{-\lambda}{2}+\frac{1}{2} \sqrt{\lambda^{2}-4 \mu} \tanh \left(\frac{1}{2} \sqrt{\lambda^{2}-4 \mu} \eta+\eta_{0}\right)\right) .
\end{aligned}
$$


Family 2 (Trigonometric Function Solutions). When $\lambda^{2}-4 \mu<0$, we obtain

$$
\begin{aligned}
v(\eta)= & a_{-1}\left(\frac{-\lambda}{2}+\frac{1}{2} \sqrt{4 \mu-\lambda^{2}} \frac{-A \sin (1 / 2) \sqrt{4 \mu-\lambda^{2}} \eta+B \cos (1 / 2) \sqrt{4 \mu-\lambda^{2}} \eta}{A \cos (1 / 2) \sqrt{4 \mu-\lambda^{2}} \eta+B \sin (1 / 2) \sqrt{4 \mu-\lambda^{2}} \eta}\right)^{-1}+a_{0} \\
& +a_{1}\left(\frac{-\lambda}{2}+\frac{1}{2} \sqrt{4 \mu-\lambda^{2}} \frac{-A \sin (1 / 2) \sqrt{4 \mu-\lambda^{2}} \eta+B \cos (1 / 2) \sqrt{4 \mu-\lambda^{2}} \eta}{A \cos (1 / 2) \sqrt{4 \mu-\lambda^{2}} \eta+B \sin (1 / 2) \sqrt{4 \mu-\lambda^{2}} \eta}\right) .
\end{aligned}
$$

Various known solutions can be rediscovered, if $A$ and $B$ are taken particular values.

For example,

(iv) if $A=0$ but $B \neq 0$, we obtain

$$
\begin{aligned}
v(\eta)= & a_{-1}\left(\frac{-\lambda}{2}+\frac{1}{2} \sqrt{4 \mu-\lambda^{2}} \cot \frac{1}{2} \sqrt{4 \mu-\lambda^{2}} \eta\right)^{-1}+a_{0} \\
& +a_{1}\left(\frac{-\lambda}{2}+\frac{1}{2} \sqrt{4 \mu-\lambda^{2}} \cot \frac{1}{2} \sqrt{4 \mu-\lambda^{2}} \eta\right)
\end{aligned}
$$

(v) if $B=0$ but $A \neq 0$, we obtain

$$
\begin{aligned}
v(\eta)= & a_{-1}\left(\frac{-\lambda}{2}-\frac{1}{2} \sqrt{4 \mu-\lambda^{2}} \tan \frac{1}{2} \sqrt{4 \mu-\lambda^{2}} \eta\right)^{-1}+a_{0} \\
& +a_{1}\left(\frac{-\lambda}{2}-\frac{1}{2} \sqrt{4 \mu-\lambda^{2}} \tan \frac{1}{2} \sqrt{4 \mu-\lambda^{2}} \eta\right)
\end{aligned}
$$

(vi) if $A \neq 0, A>B$, we obtain

$$
\begin{aligned}
v(\eta)= & a_{-1}\left(\frac{-\lambda}{2}+\frac{1}{2} \sqrt{4 \mu-\lambda^{2}} \tan \left(\frac{1}{2} \sqrt{4 \mu-\lambda^{2}} \eta-\eta_{0}\right)\right)^{-1}+a_{0} \\
& +a_{1}\left(\frac{-\lambda}{2}+\frac{1}{2} \sqrt{4 \mu-\lambda^{2}} \tan \left(\frac{1}{2} \sqrt{4 \mu-\lambda^{2}} \eta-\eta_{0}\right)\right) .
\end{aligned}
$$


Family 1 (Hyperbolic Function Solutions). Substituting (3.4) and (3.5) together with the general solution (2.5) into the (3.3), we obtain the hyperbolic function solution Equation (3.6), and then using (3.7), we obtain solutions respectively (if $A=0$ but $B \neq 0$ ),

$$
v_{1}(\eta)= \pm \sqrt{\frac{-\beta}{\gamma\left(\lambda^{2}-4 \mu\right)}}\left(-2 \lambda+\sqrt{\lambda^{2}-4 \mu} \operatorname{coth}\left(\frac{1}{2} \sqrt{\lambda^{2}-4 \mu} \eta\right)\right)
$$

where $\eta=x \pm\left(\sqrt{\left(2 \beta /\left(\lambda^{2}-4 \mu\right)\right)-\alpha}\right) t, \lambda^{2}-4 \mu \neq 0$, and $\gamma \neq 0$.

$$
v_{2}(\eta)= \pm \sqrt{\frac{-\beta}{\gamma\left(\lambda^{2}-4 \mu\right)}}\left(2 \mu\left(\frac{-\lambda}{2}+\frac{1}{2} \sqrt{\lambda^{2}-4 \mu} \operatorname{coth} \frac{1}{2} \sqrt{\lambda^{2}-4 \mu} \eta\right)^{-1}-\lambda\right)
$$

where $\eta=x \pm\left(\sqrt{\left(2 \beta /\left(\lambda^{2}-4 \mu\right)\right)-\alpha}\right) t, \lambda^{2}-4 \mu \neq 0$, and $\gamma \neq 0$.

Again, substituting (3.4) and (3.5) together with the general solution Equation (2.5) into Equation (3.3), we obtain the hyperbolic function solution Equation (3.6), and then using (3.8), our solutions become, respectively (if $B=0$ but $A \neq 0$ ),

$$
\begin{gathered}
v_{3}(\eta)= \pm \sqrt{\frac{-\beta}{\gamma\left(\lambda^{2}-4 \mu\right)}}\left(-2 \lambda+\sqrt{\lambda^{2}-4 \mu} \tanh \left(\frac{1}{2} \sqrt{\lambda^{2}-4 \mu} \eta\right)\right) . \\
v_{4}(\eta)= \pm \sqrt{\frac{-\beta}{\gamma\left(\lambda^{2}-4 \mu\right)}}\left(2 \mu\left(\frac{-\lambda}{2}+\frac{1}{2} \sqrt{\lambda^{2}-4 \mu} \tanh \left(\frac{1}{2} \sqrt{\lambda^{2}-4 \mu} \eta\right)\right)^{-1}-\lambda\right) .
\end{gathered}
$$

Also, substituting (3.4) and (3.5) together with the general solution (2.5) into the (3.3), we obtain the hyperbolic function solution (3.6), and then using (3.9), we obtain the following solutions, respectively (if $A \neq 0, A>B$ ):

$$
v_{5}(\eta)= \pm \sqrt{\frac{-\beta}{\gamma\left(\lambda^{2}-4 \mu\right)}}\left(-2 \lambda+\sqrt{\lambda^{2}-4 \mu} \tanh \left(\frac{1}{2} \sqrt{\lambda^{2}-4 \mu} \eta+\eta_{0}\right)\right)
$$

where $\eta=x \pm\left(\sqrt{\left(2 \beta /\left(\lambda^{2}-4 \mu\right)\right)-\alpha}\right) t, \lambda^{2}-4 \mu \neq 0, \gamma \neq 0$, and $\eta_{0}=\tanh ^{-1}(B / A)$. 


$$
v_{6}(\eta)= \pm \sqrt{\frac{-\beta}{\gamma\left(\lambda^{2}-4 \mu\right)}}\left(2 \mu\left(\frac{-\lambda}{2}+\frac{1}{2} \sqrt{\lambda^{2}-4 \mu} \tanh \left(\frac{1}{2} \sqrt{\lambda^{2}-4 \mu} \eta+\eta_{0}\right)\right)^{-1}-\lambda\right)
$$

where $\eta=x \pm\left(\sqrt{\left(2 \beta /\left(\lambda^{2}-4 \mu\right)\right)-\alpha}\right) t, \lambda^{2}-4 \mu \neq 0, \gamma \neq 0$, and $\eta_{0}=\tanh ^{-1}(B / A)$.

Family 2 (Trigonometric Function Solutions). Substituting (3.4) and (3.5) together with the general solution Equation (2.5) into the (3.3), we obtain the trigonometric function solution Equation (3.10), and then using (3.11), our solutions become respectively (if $A=0$ but $B \neq 0$ ),

$$
v_{7}(\eta)= \pm \sqrt{\frac{\beta}{\gamma\left(4 \mu-\lambda^{2}\right)}}\left(-2 \lambda+\sqrt{4 \mu-\lambda^{2}} \cot \left(\frac{1}{2} \sqrt{4 \mu-\lambda^{2}} \eta\right)\right)
$$

where $4 \mu-\lambda^{2} \neq 0$ and $\gamma \neq 0$.

$$
v_{8}(\eta)= \pm \sqrt{\frac{\beta}{\gamma\left(4 \mu-\lambda^{2}\right)}}\left(2 \mu\left(\frac{-\lambda}{2}+\frac{1}{2} \sqrt{4 \mu-\lambda^{2}} \cot \left(\frac{1}{2} \sqrt{4 \mu-\lambda^{2}} \eta\right)\right)^{-1}-\lambda\right)
$$

where $4 \mu-\lambda^{2} \neq 0$ and $\gamma \neq 0$.

Also, substituting (3.4) and (3.5) together with the general solution Equation (2.5) into the (3.3), we obtain the trigonometric function solution Equation (3.10), and then using (3.12), our traveling wave solutions become respectively (if $B=0$ but $A \neq 0$ ),

$$
\begin{gathered}
v_{9}(\eta)= \pm \sqrt{\frac{\beta}{\gamma\left(4 \mu-\lambda^{2}\right)}}\left(-2 \lambda-\sqrt{4 \mu-\lambda^{2}} \tan \left(\frac{1}{2} \sqrt{4 \mu-\lambda^{2}} \eta\right)\right) . \\
v_{10}(\eta)= \pm \sqrt{\frac{\beta}{\gamma\left(4 \mu-\lambda^{2}\right)}}\left(2 \mu\left(\frac{-\lambda}{2}-\frac{1}{2} \sqrt{4 \mu-\lambda^{2}} \tan \left(\frac{1}{2} \sqrt{4 \mu-\lambda^{2}} \eta\right)\right)^{-1}-\lambda\right) .
\end{gathered}
$$

Moreover, substituting (3.4) and (3.5) together with the general solution Equation (2.5) into Equation (3.3), we obtain the trigonometric function solution Equation (3.10), and then using (3.13), our obtained solutions (if $A \neq 0, A>B$ ) are as follows:

$$
v_{11}(\eta)= \pm \sqrt{\frac{\beta}{\gamma\left(4 \mu-\lambda^{2}\right)}}\left(-2 \lambda+\sqrt{4 \mu-\lambda^{2}} \tan \left(\frac{1}{2} \sqrt{4 \mu-\lambda^{2}} \eta-\eta_{0}\right)\right)
$$

where $\eta_{0}=\tan ^{-1}(B / A)$.

$$
v_{12}(\eta)= \pm \sqrt{\frac{\beta}{\gamma\left(4 \mu-\lambda^{2}\right)}}\left(2 \mu\left(\frac{-\lambda}{2}+\frac{1}{2} \sqrt{4 \mu-\lambda^{2}} \tan \left(\frac{1}{2} \sqrt{4 \mu-\lambda^{2}} \eta-\eta_{0}\right)\right)^{-1}-\lambda\right)
$$

where $\eta_{0}=\tan ^{-1}(B / A)$. 
Table 1

Zayed and Gepreel [57] solutions

Our solutions

(i) If $c_{1} \neq 0, c_{2}=0, \lambda>0$ and $\mu=0$, Equation (3.31) becomes: $u(\xi)= \pm \sqrt{(-\beta / \gamma)}(\operatorname{coth}(\lambda / 2) \xi-2)$.

(i) If $\lambda>0, \mu=0$ and $\eta=\xi$, solution $v_{1}$ becomes: $u(\xi)= \pm \sqrt{(-\beta / \gamma)}(\operatorname{coth}(\lambda / 2) \xi-2)$.

(ii) If $c_{1}=0, c_{2} \neq 0, \lambda>0$ and $\mu=0$, Equation (3.31) becomes: $u(\xi)= \pm \sqrt{(-\beta / \gamma)}(\tanh (\lambda / 2) \xi-2)$.

(ii) If $\lambda>0, \mu=0$ and $\eta=\xi$, solution $v_{3}$ becomes: $u(\xi)= \pm \sqrt{(-\beta / \gamma)}(\tanh (\lambda / 2) \xi-2)$.

(iii) If $c_{1}=0, c_{2} \neq 0, \lambda=0$ and $\mu$ is positive Equation (3.32) becomes: $u(\xi)= \pm \sqrt{(\beta / \gamma)} \cot (\sqrt{\mu} \xi)$.

(iii) If $\lambda=0, \mu$ is positive and $\eta=\xi$, solution $v_{7}$ becomes: $u(\xi)= \pm \sqrt{(\beta / \gamma)} \cot (\sqrt{\mu} \xi)$.

(iv) If $c_{1} \neq 0, c_{2}=0, \lambda=0$ and $\mu$ is positive Equation (3.32) becomes: $u(\xi)= \pm \sqrt{(\beta / \gamma)} \tan (\sqrt{\mu} \xi)$.

(iv) If $\lambda=0, \mu$ is positive and $\eta=\xi$, solution $v_{9}$ becomes: $u(\xi)= \pm \sqrt{(\beta / \gamma)} \tan (\sqrt{\mu} \xi)$.

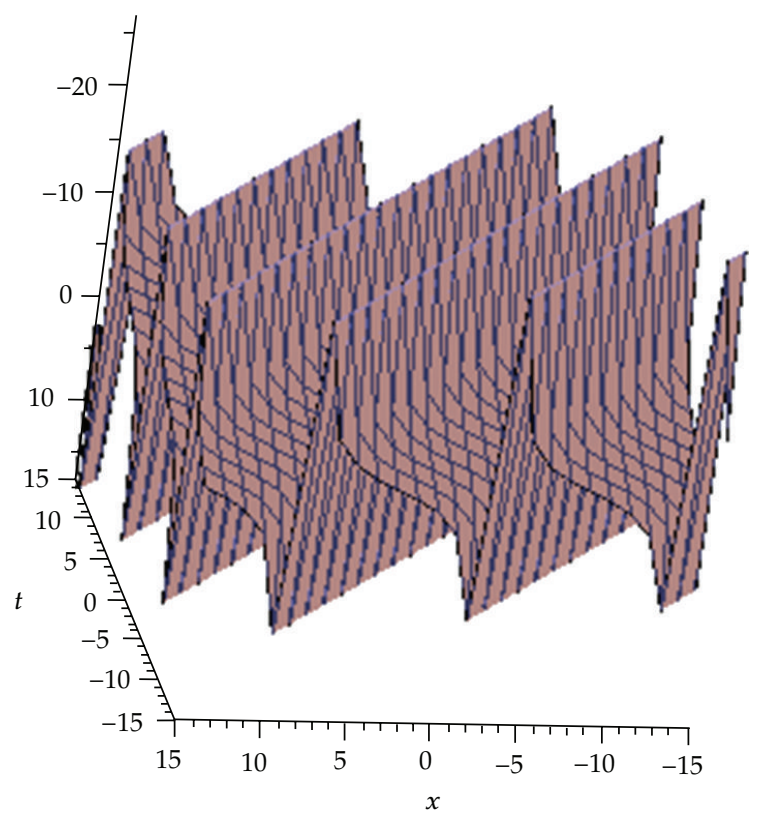

Figure 1: Periodic solution for $\alpha=3, \beta=40, \gamma=6, \lambda=4$, and $\mu=9$.

\section{Results and Discussion}

It is noteworthy to mention that some of our obtained solutions are in good agreement with the existing results which are shown in Table 1 . Furthermore, the graphical presentations of some of obtained solutions are depicted in the Figures 1, 2, 3, 4, 5, 6, 7, 8, 9, 10, and 11 . 


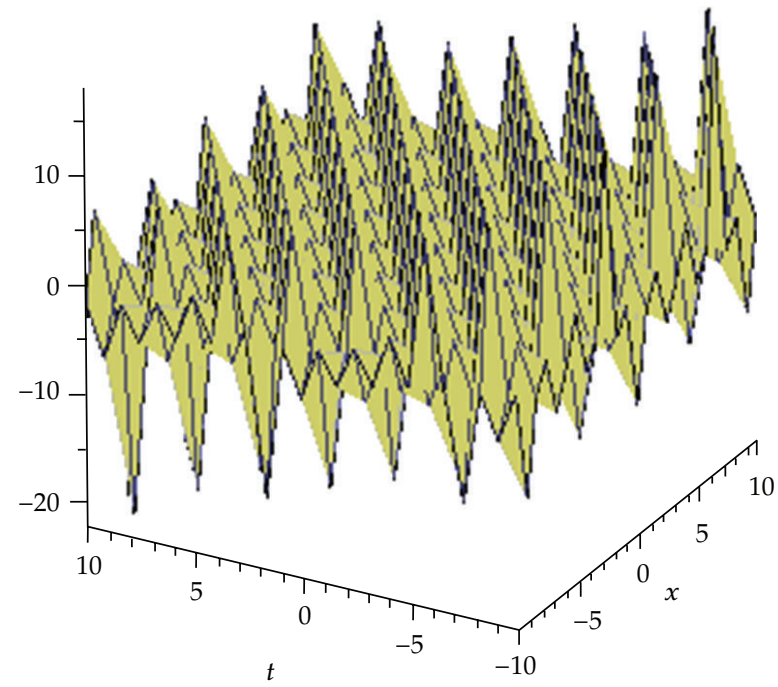

Figure 2: Periodic solution for $\alpha=1, \beta=20, \gamma=7, \lambda=3$, and $\mu=8$.

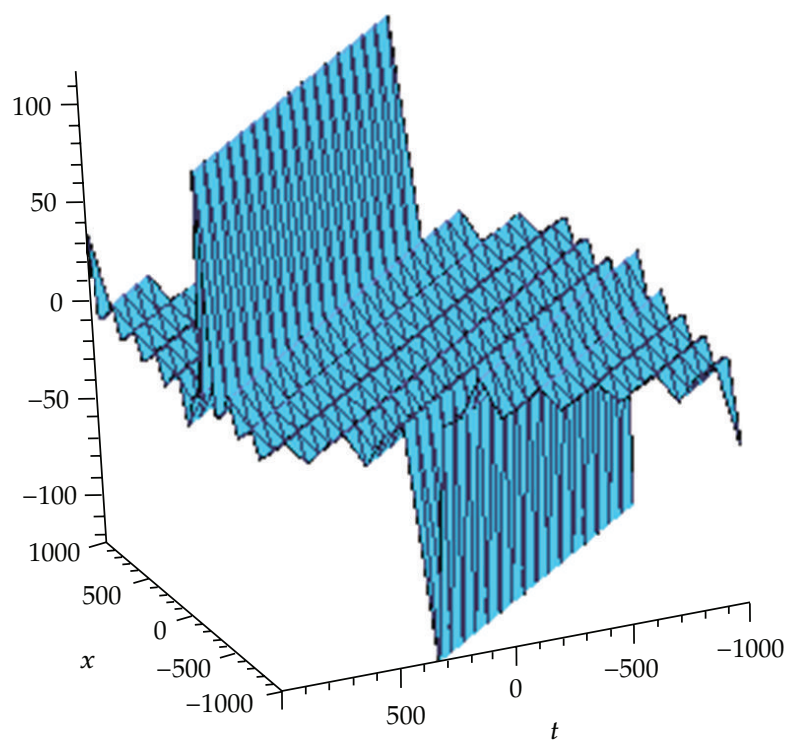

Figure 3: Periodic solution for $\alpha=3, \beta=40, \gamma=4, \lambda=2$, and $\mu=6$.

\subsection{Comparison between Zayed and Gepreel [57] Solutions and Our Solutions}

Beyond Table 1, we obtain many new exact traveling wave solutions which have not been found in the previous literature. 


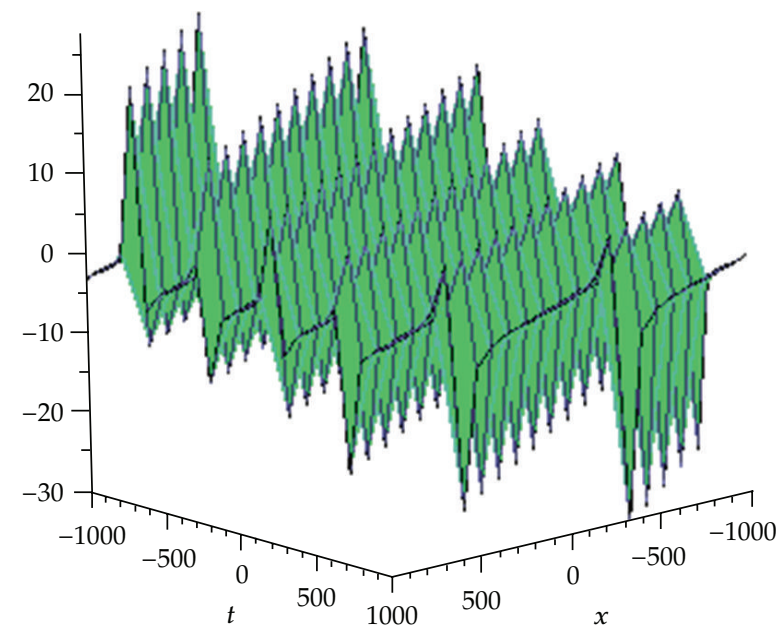

Figure 4: Solitons solution for $\alpha=1, \beta=10, \gamma=3, \lambda=1$, and $\mu=2$.

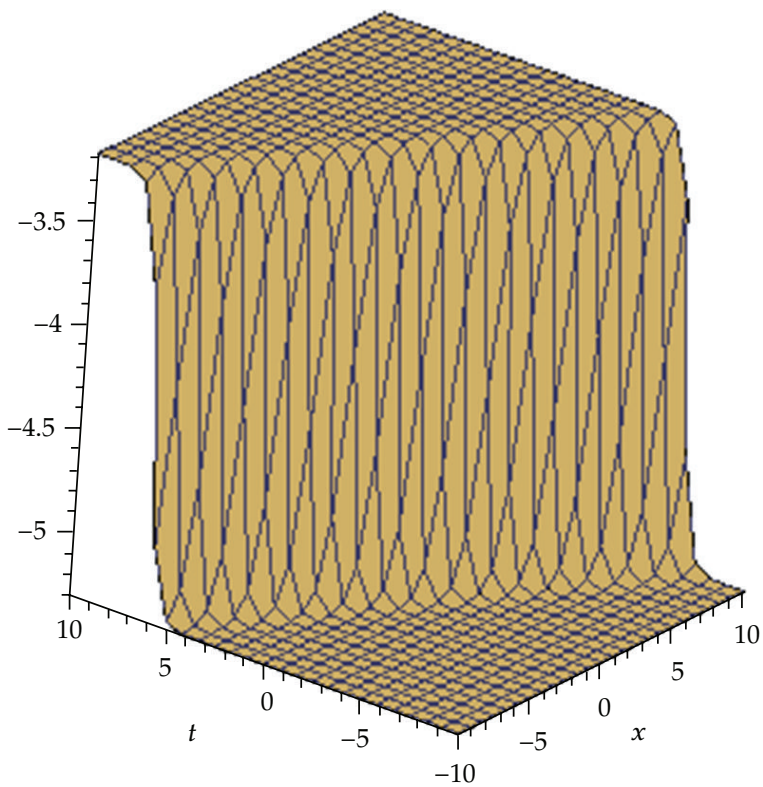

Figure 5: Periodic solution for $\alpha=2, \beta=9, \gamma=8, \lambda=4$, and $\mu=3$.

\subsection{Graphical Representations of the Solutions}

The graphical illustrations of the solutions are described in the Figures with the aid of Maple. 


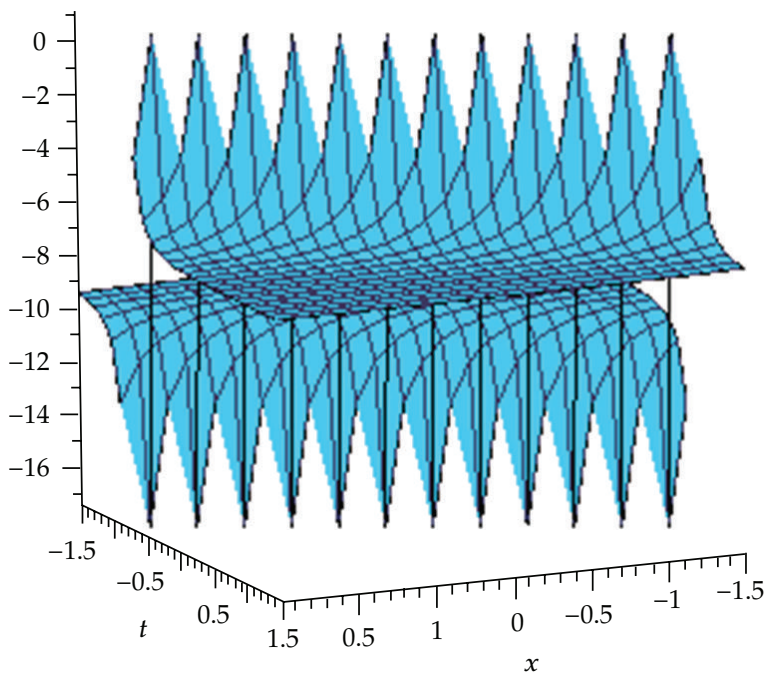

Figure 6: Periodic solution for $\alpha=2, \beta=15, \gamma=9, \lambda=7$, and $\mu=11$.

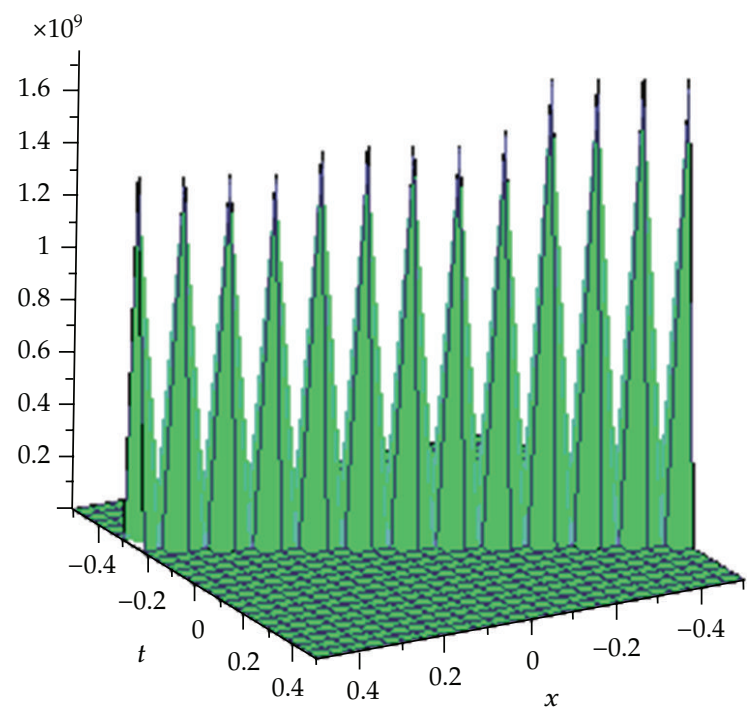

Figure 7: Solitons solution for $\alpha=2, \beta=12, \gamma=6, \lambda=6$, and $\mu=8$.

\section{Conclusions}

In this paper, we obtain abundant new exact traveling wave solutions for the nonlinear reaction diffusion equation involving parameters by applying the improved $\left(G^{\prime} / G\right)$ expansion method. The obtained solutions are expressed in terms of the hyperbolic and the trigonometric function forms. The solutions of the nonlinear reaction diffusion equation have many potential applications in biological sciences. The validity of the obtained traveling wave 


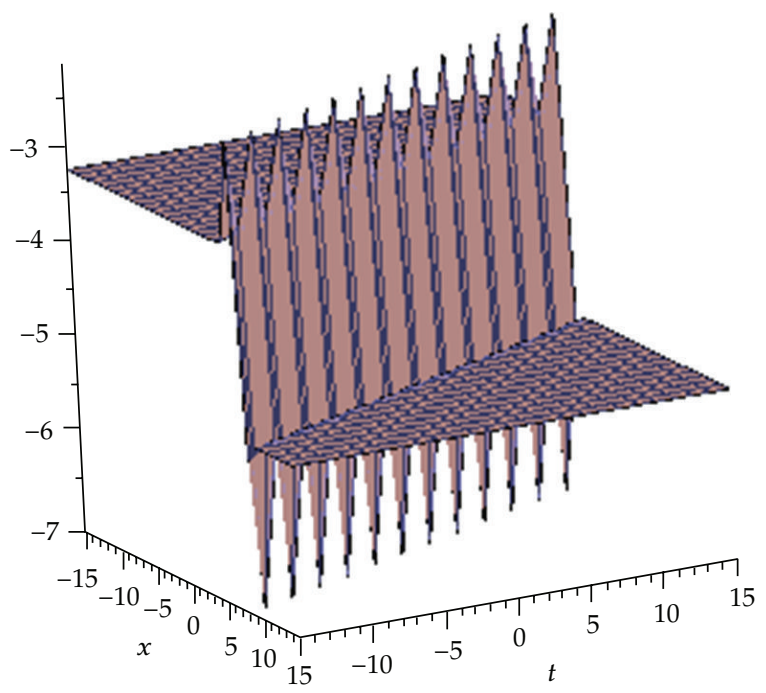

Figure 8: Solitons solution for $\alpha=2, \beta=9, \gamma=9, \lambda=6$, and $\mu=7$.

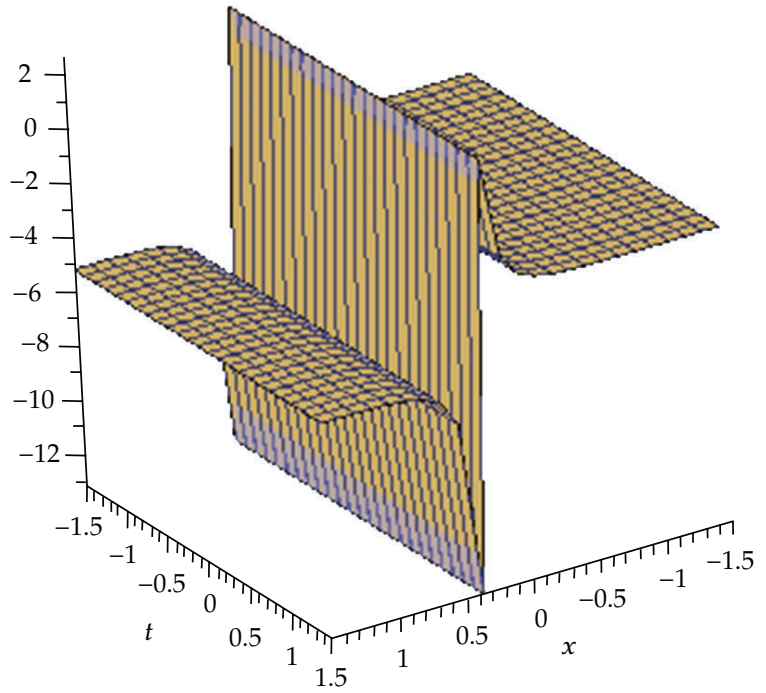

Figure 9: Periodic solution for $\alpha=1, \beta=18, \gamma=9, \lambda=8$, and $\mu=7$.

solutions is proved by comparing with the published results. We expect that the used method will be effectively used to construct many new exact traveling wave solutions for other kinds of nonlinear evolution equations which are arising in technical arena. 


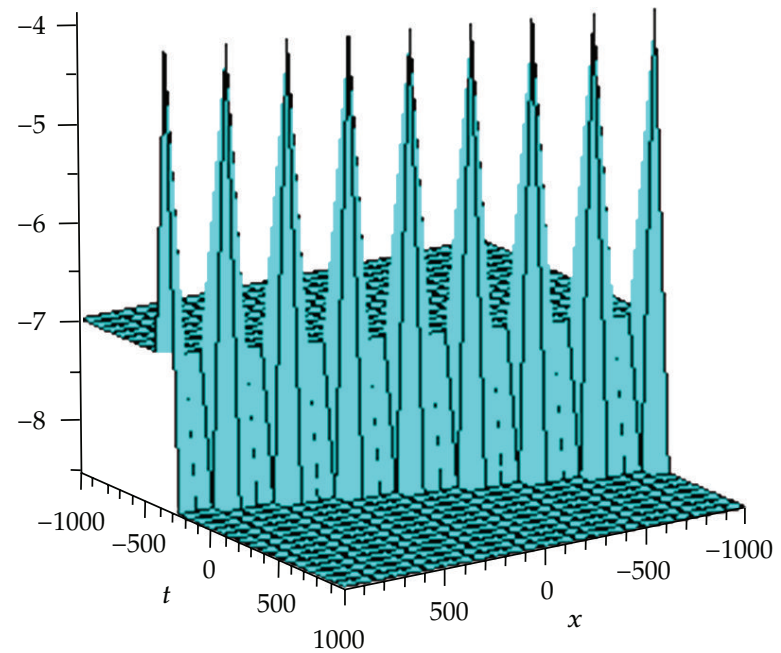

Figure 10: Solitons solution for $\alpha=3, \beta=6, \gamma=10, \lambda=5$, and $\mu=6$.

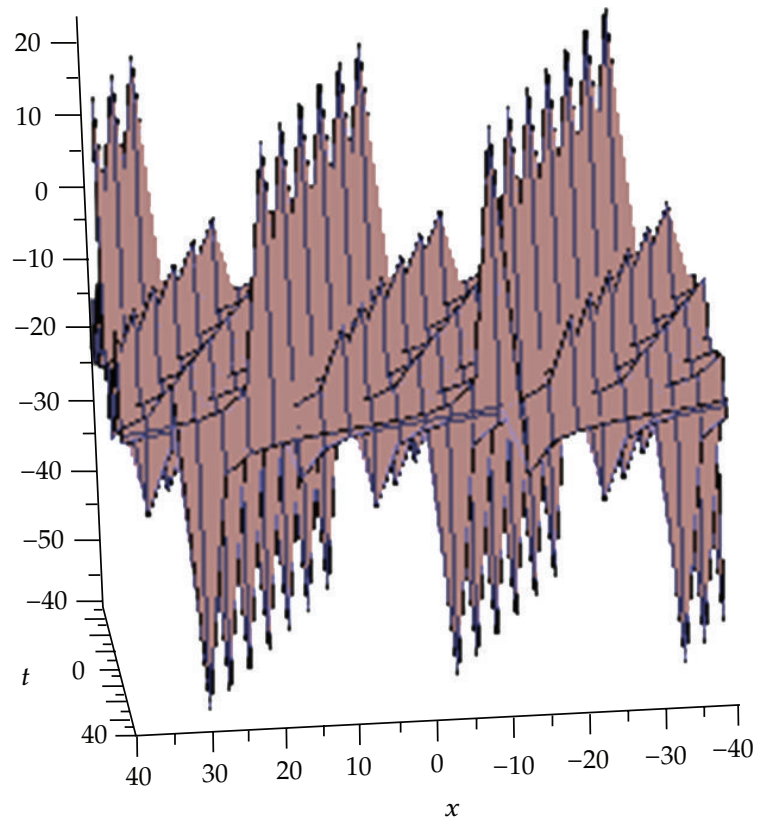

Figure 11: Solitons solution for $\alpha=2, \beta=22, \gamma=8, \lambda=9$, and $\mu=21$.

\section{Acknowledgments}

This paper is supported by the USM short-term grant (reference no. 304/PMATHS/6310072) and the authors would like to express their thanks to the School of Mathematical Sciences, USM, for providing related research facilities. The authors are also grateful to the referee(s) for their valuable comments and suggestions. 


\section{References}

[1] K. W. Chow, "A class of exact, periodic solutions of nonlinear envelope equations," Journal of Mathematical Physics, vol. 36, no. 8, pp. 4125-4137, 1995.

[2] S. Liu, Z. Fu, S. Liu, and Q. Zhao, "Jacobi elliptic function expansion method and periodic wave solutions of nonlinear wave equations," Physics Letters A, vol. 289, no. 1-2, pp. 69-74, 2001.

[3] R. Hirota, "Exact solution of the korteweg-de vries equation for multiple collisions of solitons," Physical Review Letters, vol. 27, no. 18, pp. 1192-1194, 1971.

[4] M. Wang and X. Li, "Applications of F-expansion to periodic wave solutions for a new Hamiltonian amplitude equation," Chaos, Solitons and Fractals, vol. 24, no. 5, pp. 1257-1268, 2005.

[5] C. Rogers and W. F. Shadwick, Bäcklund Transformations and Their Applications, vol. 161 of Mathematics in Science and Engineering, Academic Press, New York, NY, USA, 1982.

[6] L. Jianming, D. Jie, and Y. Wenjun, "Bäcklund transformation and new exact solutions of the SharmaTasso-Olver equation," Abstract and Applied Analysis, vol. 2011, Article ID 935710, 8 pages, 2011.

[7] Z. Yan and H. Zhang, "New explicit solitary wave solutions and periodic wave solutions for Whitham-Broer-Kaup equation in shallow water," Physics Letters A, vol. 285, no. 5-6, pp. 355-362, 2001.

[8] C. A. Gómez, A. H. Salas, and B. Acevedo Frias, "Exact solutions to KdV6 equation by using a new approach of the projective Riccati equation method," Mathematical Problems in Engineering, vol. 2010, Article ID 797084, 10 pages, 2010.

[9] X. Li and M. Wang, "A sub-ODE method for finding exact solutions of a generalized KdV-mKdV equation with high-order nonlinear terms," Physics Letters A, vol. 361, no. 1-2, pp. 115-118, 2007.

[10] M. Wang, Y. Zhou, and Z. Li, "Application of a homogeneous balance method to exact solutions of nonlinear equations in mathematical physics," Physics Letters A, vol. 216, no. 1-5, pp. 67-75, 1996.

[11] E. M. E. Zayed, H. A. Zedan, and K. A. Gepreel, "On the solitary wave solutions for nonlinear HirotaSatsuma coupled KdV of equations," Chaos, Solitons and Fractals, vol. 22, no. 2, pp. 285-303, 2004.

[12] A.-M. Wazwaz, "The tanh-coth method for solitons and kink solutions for nonlinear parabolic equations," Applied Mathematics and Computation, vol. 188, no. 2, pp. 1467-1475, 2007.

[13] E. Yusufoğlu and A. Bekir, "Exact solutions of coupled nonlinear Klein-Gordon equations," Mathematical and Computer Modelling, vol. 48, no. 11-12, pp. 1694-1700, 2008.

[14] A. Bekir and A. C. Cevikel, "Solitary wave solutions of two nonlinear physical models by tanh-coth method," Communications in Nonlinear Science and Numerical Simulation, vol. 14, no. 5, pp. 1804-1809, 2009.

[15] F. Taşcan and A. Bekir, "Analytic solutions of the (2+1)-dimensional nonlinear evolution equations using the sine-cosine method," Applied Mathematics and Computation, vol. 215, no. 8, pp. 3134-3139, 2009.

[16] F. Taşcan and A. Bekir, "Applications of the first integral method to nonlinear evolution equations," Chinese Physics B, vol. 19, no. 8, Article ID 080201, 2010.

[17] A. H. Salas and C. A. Gómez S., "Application of the Cole-Hopf transformation for finding exact solutions to several forms of the seventh-order KdV equation," Mathematical Problems in Engineering, vol. 2010, Article ID 194329, 14 pages, 2010.

[18] J.-H. He and X.-H. Wu, "Exp-function method for nonlinear wave equations," Chaos, Solitons and Fractals, vol. 30, no. 3, pp. 700-708, 2006.

[19] S. T. Mohyud-Din, M. A. Noor, and K. I. Noor, "Exp-function method for traveling wave solutions of modified zakharov-kuznetsov equation," Journal of King Saud University, vol. 22, no. 4, pp. 213-216, 2010.

[20] A. Bekir, "The exp-function method for ostrovsky equation," International Journal of Nonlinear Sciences and Numerical Simulation, vol. 10, no. 6, pp. 735-739, 2009.

[21] A. Yıldırım and Z. Pınar, "Application of the exp-function method for solving nonlinear reactiondiffusion equations arising in mathematical biology," Computers \& Mathematics with Applications, vol. 60, no. 7, pp. 1873-1880, 2010.

[22] H. Naher, F. A. Abdullah, and M. A. Akbar, "New traveling wave solutions of the higher dimensional nonlinear partial differential equation by the Exp-function method," Journal of Applied Mathematics, vol. 2012, Article ID 575387, 14 pages, 2012.

[23] H. Naher, F. A. Abdullah, and M. A. Akbar, "The exp-function method for new exact solutions of the nonlinear partial differential equations," International Journal of the Physical Sciences, vol. 6, no. 29, pp. 6706-6716, 2011. 
[24] W. Zhang, "The extended tanh method and the exp-function method to solve a kind of nonlinear heat equation," Mathematical Problems in Engineering, vol. 2010, Article ID 935873, 12 pages, 2010.

[25] İ. Aslan, "Application of the exp-function method to nonlinear lattice differential equations for multiwave and rational solutions," Mathematical Methods in the Applied Sciences, vol. 34, no. 14, pp. 17071710, 2011.

[26] A. M. Wazwaz, "A new (2+1)-dimensional Korteweg-de-Vries equation and its extension to a new (3+1)-dimensional Kadomtsev-Petviashvili equation," Physica Scripta, vol. 84, no. 3, Article ID 035010, 2011.

[27] X. Liu, L. Tian, and Y. Wu, "Exact solutions of the generalized Benjamin-Bona-Mahony equation," Mathematical Problems in Engineering, vol. 2010, Article ID 796398, 5 pages, 2010.

[28] A. Biswas, C. Zony, and E. Zerrad, "Soliton perturbation theory for the quadratic nonlinear KleinGordon equation," Applied Mathematics and Computation, vol. 203, no. 1, pp. 153-156, 2008.

[29] R. Sassaman and A. Biswas, "Soliton perturbation theory for phi-four model and nonlinear KleinGordon equations," Communications in Nonlinear Science and Numerical Simulation, vol. 14, no. 8, pp. 3239-3249, 2009.

[30] R. Sassaman and A. Biswas, "Topological and non-topological solitons of the generalized KleinGordon equations," Applied Mathematics and Computation, vol. 215, no. 1, pp. 212-220, 2009.

[31] R. Sassaman, A. Heidari, and A. Biswas, "Topological and non-topological solitons of nonlinear KleinGordon equations by He's semi-inverse variational principle," Journal of the Franklin Institute, vol. 347, no. 7, pp. 1148-1157, 2010.

[32] R. Sassaman, A. Heidari, F. Majid, E. Zerrad, and A. Biswas, “Topological and non-topological solitons of the generalized Klein-Gordon equations in 1+2 dimensions," Dynamics of Continuous, Discrete $\mathcal{E}$ Impulsive Systems A, vol. 17, no. 2, pp. 275-286, 2010.

[33] R. Sassaman and A. Biswas, "Topological and non-topological solitons of the Klein-Gordon equations in 1+2 dimensions," Nonlinear Dynamics, vol. 61, no. 1-2, pp. 23-28, 2010.

[34] R. Sassaman, M. Edwards, F. Majid, and A. Biswas, "1-Soliton solution of the coupled nonlinear KlienGordon equations," Studies in Mathematical Sciences, vol. 1, no. 1, pp. 30-37, 2010.

[35] R. Sassaman and A. Biswas, "Soliton solution of the generalized Klien-Gordon equation by semiinverse variational principle," Mathematics in Engineering, Science and Aerospace, vol. 2, no. 1, pp. 99 104, 2011.

[36] R. Sassaman and A. Biswas, "1-Soliton solution of the Perturbed Klien-Gordon equation," Physics Express, vol. 1, no. 1, pp. 9-14, 2011.

[37] A. Kiliçman and H. Eltayeb, "On a new integral transform and differential equations," Mathematical Problems in Engineering, vol. 2010, Article ID 463579, 13 pages, 2010.

[38] M. A. Noor, K. I. Noor, E. Al-Said, and M. Waseem, "Some new iterative methods for nonlinear equations," Mathematical Problems in Engineering, vol. 2010, Article ID 198943, 12 pages, 2010.

[39] E. G. Bakhoum and C. Toma, "Mathematical transform of traveling-wave equations and phase aspects of quantum interaction," Mathematical Problems in Engineering, vol. 2010, Article ID 695208, 15 pages, 2010.

[40] M. A. Abdou, "The extended F-expansion method and its application for a class of nonlinear evolution equations," Chaos, Solitons and Fractals, vol. 31, no. 1, pp. 95-104, 2007.

[41] A. S. Deakin and M. Davison, "An analytic solution for a Vasicek interest rate convertible bond model," Journal of Applied Mathematics, vol. 2010, Article ID 263451, 5 pages, 2010.

[42] P. Sunthrayuth and P. Kumam, "A new general iterative method for solution of a new general system of variational inclusions for nonexpansive semigroups in Banach spaces," Journal of Applied Mathematics, vol. 2011, Article ID 187052, 29 pages, 2011.

[43] B. I. Yun, "An iteration method generating analytical solutions for Blasius problem," Journal of Applied Mathematics, vol. 2011, Article ID 925649, 8 pages, 2011.

[44] M. Wang, X. Li, and J. Zhang, "The (G'/G)-expansion method and travelling wave solutions of nonlinear evolution equations in mathematical physics," Physics Letters. A, vol. 372, no. 4, pp. 417423, 2008.

[45] A. Malik, F. Chand, and S. C. Mishra, "Exact travelling wave solutions of some nonlinear equations by (G'/G)-expansion method," Applied Mathematics and Computation, vol. 216, no. 9, pp. 2596-2612, 2010.

[46] A. Bekir, "Application of the (G'/G)-expansion method for nonlinear evolution equations," Physics Letters A, vol. 372, no. 19, pp. 3400-3406, 2008. 
[47] E. M. E. Zayed, "New traveling wave solutions for higher dimensional nonlinear evolution equations using a generalized $\left(\mathrm{G}^{\prime} / \mathrm{G}\right)$-expansion method," Journal of Applied Mathematics $\mathcal{E}$ Informatics, vol. 28, no. 1-2, pp. 383-395, 2010.

[48] H. Naher, F. A. Abdullah, and M. A. Akbar, "The ( $\left.\mathrm{G}^{\prime} / \mathrm{G}\right)$-expansion method for abundant traveling wave solutions of Caudrey-Dodd-Gibbon equation," Mathematical Problems in Engineering, vol. 2011, Article ID 218216, 11 pages, 2011.

[49] M. Hayek, "Constructing of exact solutions to the $\mathrm{KdV}$ and Burgers equations with power-law nonlinearity by the extended $\left(\mathrm{G}^{\prime} / \mathrm{G}\right)$-expansion method," Applied Mathematics and Computation, vol. 217, no. 1, pp. 212-221, 2010.

[50] S. Guo and Y. Zhou, "The extended (G'/G)-expansion method and its applications to the WhithamBroer-Kaup-like equations and coupled Hirota-Satsuma KdV equations," Applied Mathematics and Computation, vol. 215, no. 9, pp. 3214-3221, 2010.

[51] E. M. E. Zayed and S. Al-Joudi, “Applications of an extended (G'/G)-expansion method to find exact solutions of nonlinear PDEs in mathematical physics," Mathematical Problems in Engineering, vol. 2010, Article ID 768573, 19 pages, 2010.

[52] J. Zhang, F. Jiang, and X. Zhao, "An improved ( $\left.\mathrm{G}^{\prime} / \mathrm{G}\right)$-expansion method for solving nonlinear evolution equations," International Journal of Computer Mathematics, vol. 87, no. 8, pp. 1716-1725, 2010.

[53] Y. S. Hamad, M. Sayed, S. K. Elagan, and E. R. El-Zahar, "The improved (G'/G)-expansion method for solving (3+1)-dimensional potential-YTSF equation," Journal of Modern Methods in Numerical Mathematics, vol. 2, no. 1-2, pp. 32-38, 2011.

[54] T. A. Nofel, M. Sayed, Y. S. Hamad, and S. K. Elagan, "The improved (G'/G)-expansion method for solving the fifth-order KdV equation," Annals of Fuzzy Mathematics and Informatics, vol. 3, no. 1, pp. 9-17, 2012.

[55] Y. M. Zhao, Y. J. Yang, and W. Li, "Application of the improved (G'/G)-expansion method for the variant Boussinesq equations," Applied Mathematical Sciences, vol. 5, no. 58, pp. 2855-2861, 2011.

[56] S. Tao and T. Xia, "An improved ( $\left.\mathrm{G}^{\prime} / \mathrm{G}\right)$ - expansion method and its application to the $(3+1)-$ dimensional kdv equation," in International Conference on Information Science and Technology (ICIST '11), pp. 280-286, March 2011.

[57] E. M. E. Zayed and K. A. Gepreel, "The $\left(\mathrm{G}^{\prime} / \mathrm{G}\right)$-expansion method for finding traveling wave solutions of nonlinear partial differential equations in mathematical physics," Journal of Mathematical Physics, vol. 50, no. 1, Article ID 013502, 12 pages, 2009. 


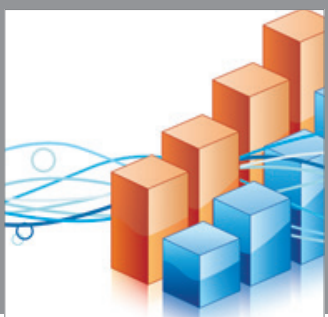

Advances in

Operations Research

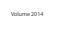

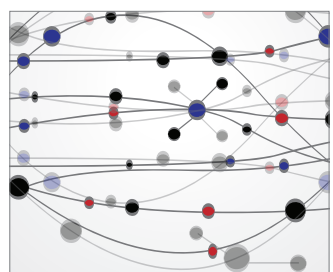

\section{The Scientific} World Journal
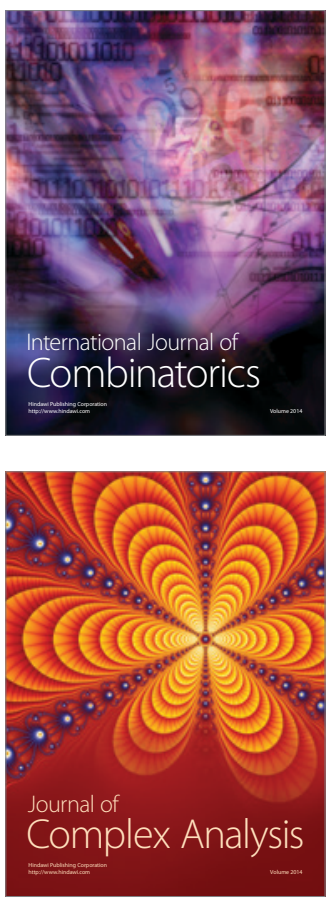

International Journal of

Mathematics and

Mathematical

Sciences
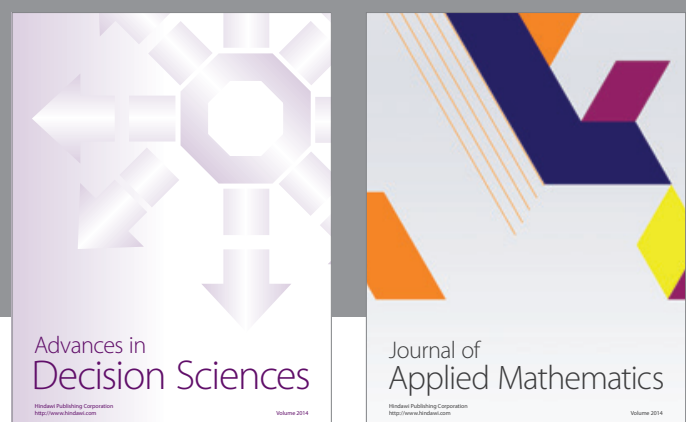

Journal of

Applied Mathematics
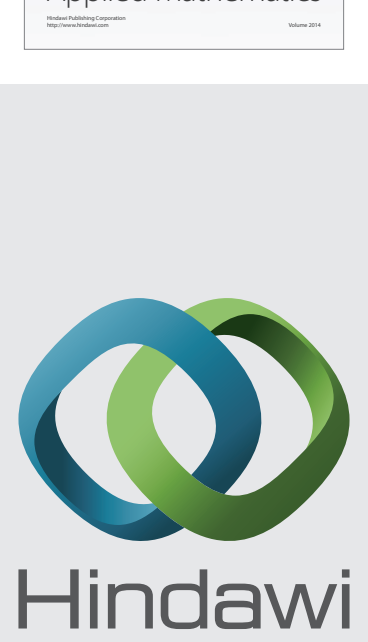

Submit your manuscripts at http://www.hindawi.com
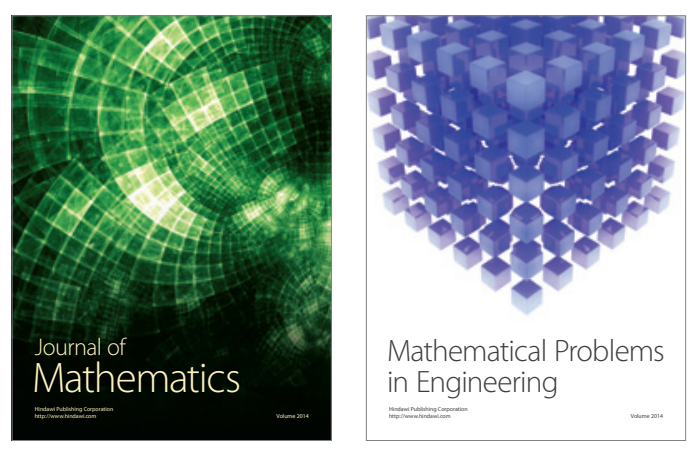

Mathematical Problems in Engineering
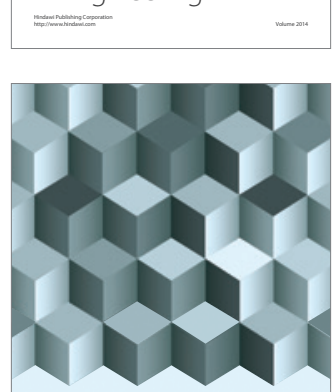

Journal of

Function Spaces
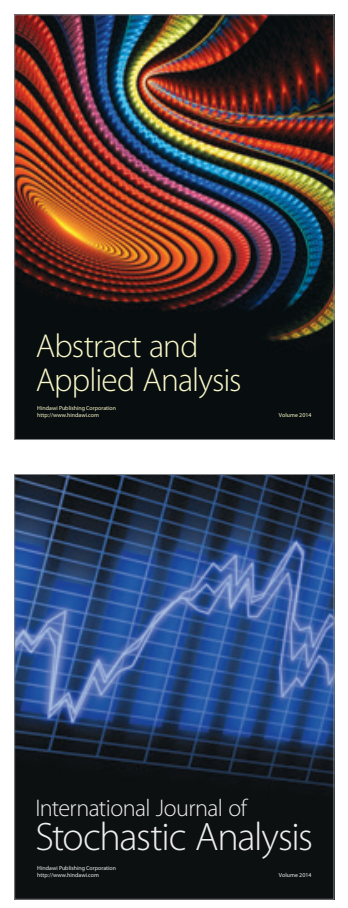

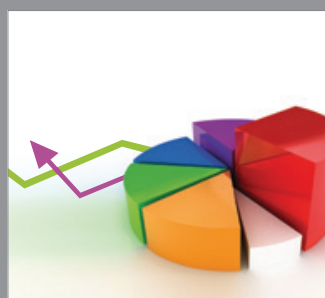

ournal of

Probability and Statistics

Promensencen
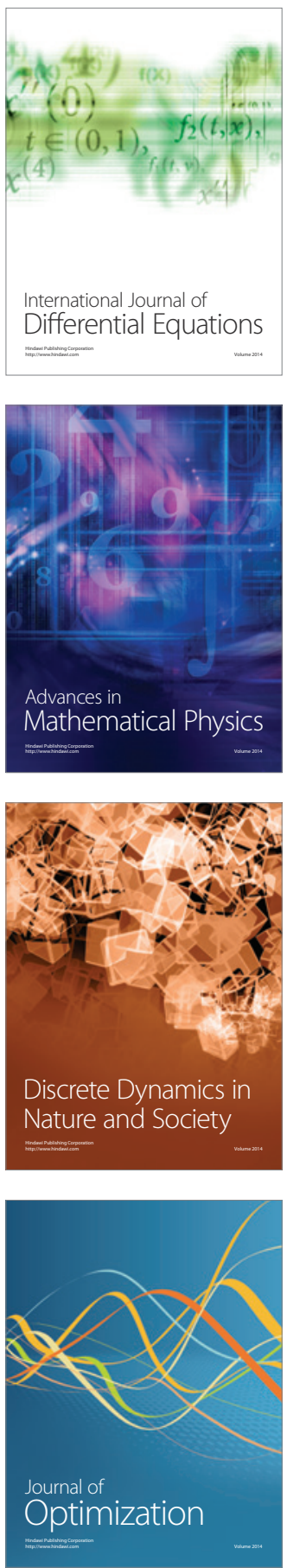